\title{
ZnO Based Charge Trapping Memory with Embedded Nanoparticles
}

\author{
Ayman Rizk, Feyza B. Oruç, Ali K. Okyay, Member IEEE and Ammar Nayfeh, Member IEEE
}

\begin{abstract}
A thin film $\mathrm{ZnO}$ charge trapping memory cell with embedded nanoparticles is demonstrated by Physics Based TCAD simulation. The results show $3 \mathrm{~V}$ increase in the $V_{t}$ shift due to the nanoparticles for the same operating voltage. In addition $a \sim 6 \mathrm{~V}$ reduction in the programming voltage is obtained due the nanoparticles. In addition, the effect of the trapping layer and tunnel oxide scaling on the $\mathbf{1 0}$ year retention time is studied.
\end{abstract}

Index Terms - ZnO, Memory, Nanoparticles, Charge Trapping, Nano

\section{INTRODUCTION}

With the current trend of super handheld computing devices like the iPad, low power memory devices are necessary. Research has commenced to determine if nanotechnology can be utilized to achieve this goal. This includes the use of nanowires or nanoparticles to enhance the performance of memory devices [1-4].

Recently, $\mathrm{ZnO}$ metal-oxide semiconductors have been extensively investigated as channel materials for thin film transistors (TFTs). As a result, memory devices based on $\mathrm{ZnO}$ is of interest [5-8]. In this work we demonstrate by TCAD a nonvolatile memory device with a $\mathrm{ZnO}$ film as the charge-trapping layer in addition to $\mathrm{ZnO}$ channel material with embedded nanoparticles that reduces the operating voltage.

\section{TCAD STRUCTURE AND MODEL}

Figure 1 shows the basic structure of the memory cell simulated using the Synopsys ${ }^{\mathrm{TM}}$ TCAD tools. A Physics Based TCAD approach is used with key parameters modified to account for the new materials highlighted in table 1 [9-15]. The structure is stack of $\mathrm{ZnO}$ (channel) followed by a $5 \mathrm{~nm} \mathrm{Al}_{2} \mathrm{O}_{3}$ (tunnel oxide), $2 \mathrm{~nm} \mathrm{ZnO}$ (trapping layer) and $15 \mathrm{~nm}$ of $\mathrm{Al}_{2} \mathrm{O}_{3}$ as the blocking oxide.

Manuscript received July 4, 2012. This work was supported by the Masdar Institute of Science and Technology

F. B. Oruc is with the Department of Electrical and Electronics Engineering, Bilkent University, Ankara 06800, Turkey

A. K. Okyay is with the Department of Electrical and Electronics Engineering and UNAM-Institute of Materials Science and

Nanotechnology, Bilkent University, Ankara 06800, Turkey (phone: +90532-5456351; fax: +90-312-2664192; e-mail: aokyay@ee.bilkent.edu.tr).

A. Rizk is with Masdar Institute of Science and Technology Abu Dhabi, United Arab Emirates

A. Nayfeh is with Microsystems Engineering at Masdar Institute of Science and Technology Abu Dhabi, PO BOX 54224 United Arab Emirates; (phone: +97128109105; e-mail: anayfeh $@$ masdar.ac.ae).

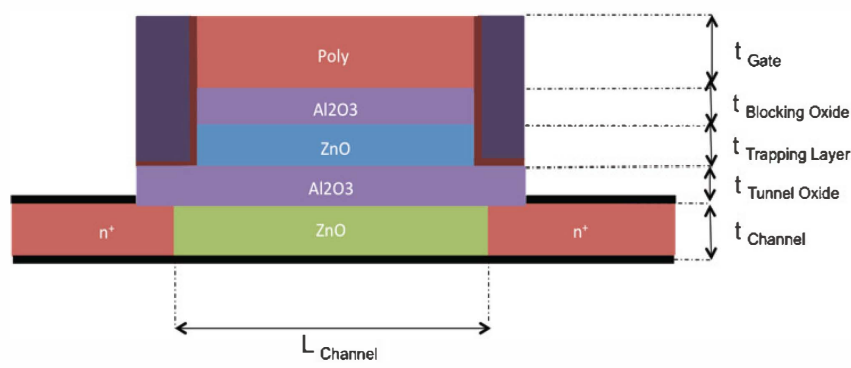

Figure 1: Cross-section of the simulated memory cell

\begin{tabular}{lll|ll} 
& $\mathbf{A l}_{2} \mathbf{O}_{3}$ & $\mathbf{S i O}_{2}$ & $\mathbf{Z n O}$ & $\mathbf{S i}_{3} \mathbf{N}_{4}$ \\
\hline Relative Permittivity & $9.5(6$ to 9$)$ & 3.9 & 8.75 & 7.5 \\
\hline Band Gap & $6.65 \mathrm{eV}$ & $9 \mathrm{eV}$ & $3.37 \mathrm{eV}$ & $5 \mathrm{eV}$ \\
\hline Electron Affinity & $2.58 \mathrm{eV}$ & $0.9 \mathrm{eV}$ & $4.5 \mathrm{eV}$ & $1.9 \mathrm{eV}$ \\
\hline Electron Tunnel Mass & $0.43 \mathrm{~m}_{0}$ & $0.44 \mathrm{~m}_{0}$ & $0.24 \mathrm{~m}_{0}$ & $0.36 \mathrm{~m}_{0}$ \\
\hline Hole Tunnel Mass & $0.5 \mathrm{~m}_{0}$ & $1 \mathrm{~m}_{0}$ & $0.59 \mathrm{~m}_{0}$ & $0.38 \mathrm{~m}_{0}$
\end{tabular}

Table 1: Physics Based TCAD material parameters

Figure 2 is the band diagram of the structure with zero applied voltage. The diagram shows a quantum well between blocking and tunnel oxides in the trapping layer due to the band offsets. Electrons can tunnel from the $\mathrm{ZnO}$ substrate to this potential well within the trapping layer. By adding nanopartciles embedded in the trapping layer additional energy levels will be availble for the electrons to tunnel into thus increasing the memroy effect.

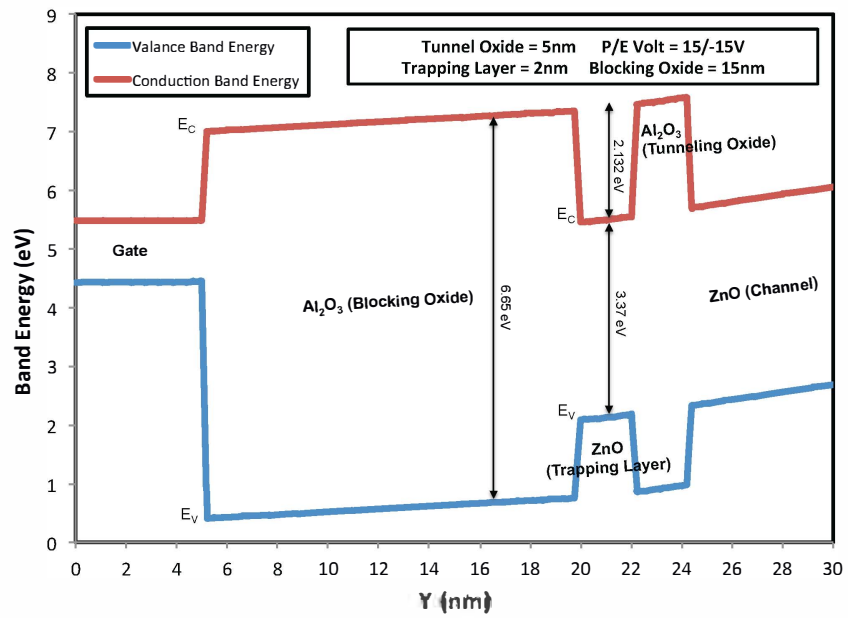

Figure 2: Conduction and valance band energy across the device $(\mathrm{V}=0)$ 


\section{MEMORY EFFECT}

In order to test the functionality of the memory cell the threshold voltage is measured after programing and erasing. The program and erase states of the device are simulated for several cycles to achieve a stead-state charge density and then the $I_{d}-V_{g}$ curves are simulated to extract the corresponding threshold voltages. During each cycle, both the drain and source are set to zero voltage then a $2.5 \mathrm{~ms}$ wide positive pulse $(15 \mathrm{~V})$ is applied to the gate during the programming cycle followed by a $5 \mathrm{~ms}$ wide negative pulse $(-15 \mathrm{~V})$ during the erase cycle. Each pulse is separated by $2.5 \mathrm{~ms}$. Throughout the program and erase cycles, the electrons and holes can tunnel between from the $\mathrm{ZnO}$ channel to the trapping layer channel. The charge distribution established in the program state is mainly due to electrons tunneling into the $\mathrm{ZnO}$ trapping layer from the channel. During the erase cycle when the negative bias is applied to the gate, electrons will tunnel out of the $\mathrm{ZnO}$ trapping layer and into the channel, but the majority of the tunneling comes from holes that tunnel into the $\mathrm{ZnO}$ trapping layer from the channel. When inside the trapping layer they then recombine with stored electrons.

Figure 3 plots the $I_{d}-V_{g}$ curve of both the programed and erase states using $15 \mathrm{~V} /-15 \mathrm{~V}$ respectively for a memory cell with parameters in figure 2 . In this case the $V_{t}$ shift is only around $0.346 \mathrm{~V}$. This is due to the fact that the electrons that tunnel across the $\mathrm{Al}_{2} \mathrm{O}_{3}$ tunnel oxide can only be trapped in a quantum well of around $2 \mathrm{eV}$ due to the conduction band offsets between $\mathrm{ZnO}$ and $\mathrm{Al}_{2} \mathrm{O}_{3}$. Additional available quantum states in the $\mathrm{ZnO}$ layer are needed to allow for more electron trapping and hence a larger $V_{t}$ shift.

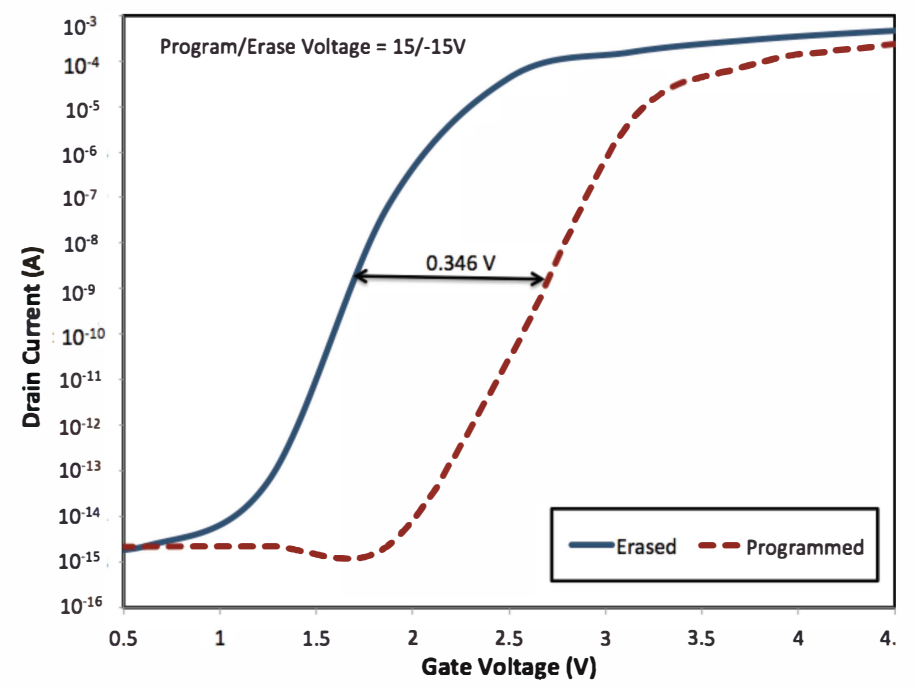

Figure 3: $I_{d}-V g$ with and without nanoparticles

\section{EFFECT OF NANOPARTICLES}

\section{a. VT SHIFT}

In order to study the effect to adding nanoparticles in the $\mathrm{ZnO}$ trapping layer additional states are added at different energy levels. Figure 4 shows the band diagram of the $\mathrm{ZnO}$ layer with added quantum states located at $\mathrm{E}_{\mathrm{c}}-\mathrm{E}_{\text {trap }}=1 \mathrm{eV}$ (donor state) and $\mathrm{E}_{\mathrm{c}}-\mathrm{E}_{\text {trap }}=2.5 \mathrm{eV}$ (acceptor) with density of $10^{20} \mathrm{~cm}^{-3}$. The values were taken from previously studied $\mathrm{Si}_{3} \mathrm{~N}_{4}$ SONOS memory [16-17].
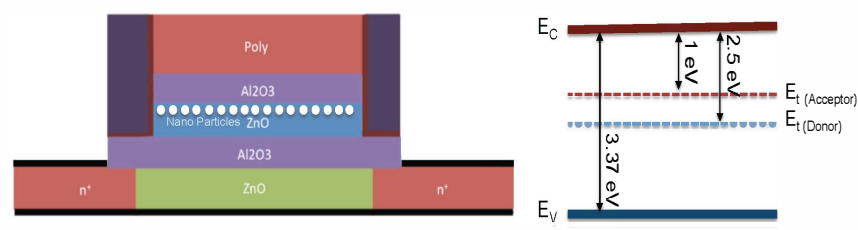

Figure: 4 Left: Device structure with the nanoparticle within the trapping layer. Right: The band diagram of the $\mathrm{ZnO}$ layer with added quantum states

In addition the density of nanoparticles is also studied. The simulation includes a density of $1 \times 10^{10} / \mathrm{cm}^{3}$ and $1 \times 10^{20} / \mathrm{cm}^{3}$. Figure 5 plots the $\mathrm{I}_{\mathrm{d}}-\mathrm{V}_{\mathrm{g}}$ curve of both the programed and erase states using $15 \mathrm{~V} /-15 \mathrm{~V}$ respectively for a memory cell with parameters in figure 2 with and without nanoparticles. The $\mathrm{V}_{\mathrm{t}}$ shift increases from $0.346 \mathrm{~V}$ to $2.1 \mathrm{~V}$ and $3.4 \mathrm{~V}$ for $1 \times 10^{10} / \mathrm{cm}^{3}$ and $1 \times 10^{20} / \mathrm{cm}^{3}$ density of nanoparticles respectively for the same programing voltage applied. The nanoparticles increase the amount of tunneling for the same voltage applied and thus the $V_{t}$ shift increases.

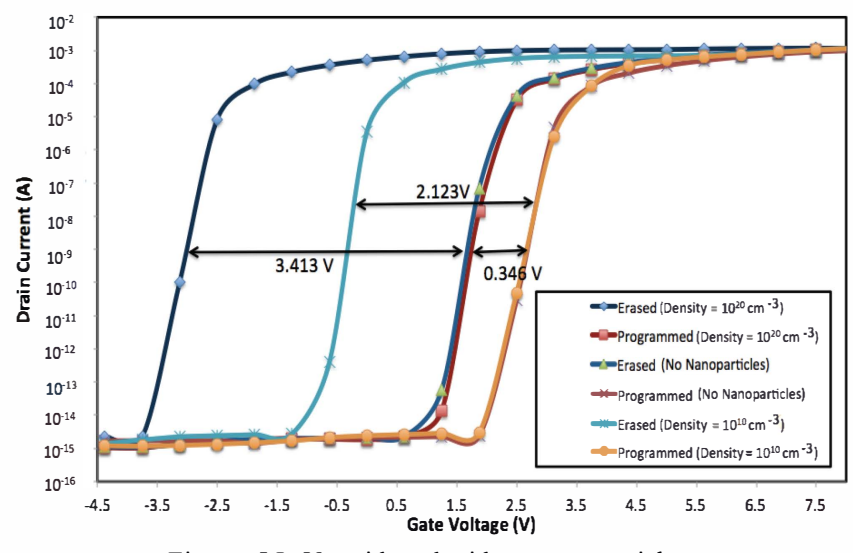

Figure: $5 \mathrm{I}_{\mathrm{d}}-\mathrm{Vg}$ with and without nanoparticles.

\section{b. Programming Voltage}

In addition to affecting the amount of $\mathrm{V}_{\mathrm{t}}$ shift, the nanoparticles also affect the programming voltage needed. Figure 6 shows the threshold voltage shift vs. programing voltage with and without the nanoparticles showing the reduction in programing voltage. For a $3 \mathrm{~V} \mathrm{~V}_{\mathrm{t}}$ shift the programming voltage reduces from $18 \mathrm{~V}$ to $12.5 \mathrm{~V}$. It should be noted, the nanoparticles can be spin coated between separate ZnO Atomic Layer Deposition (ALD) growth steps. 


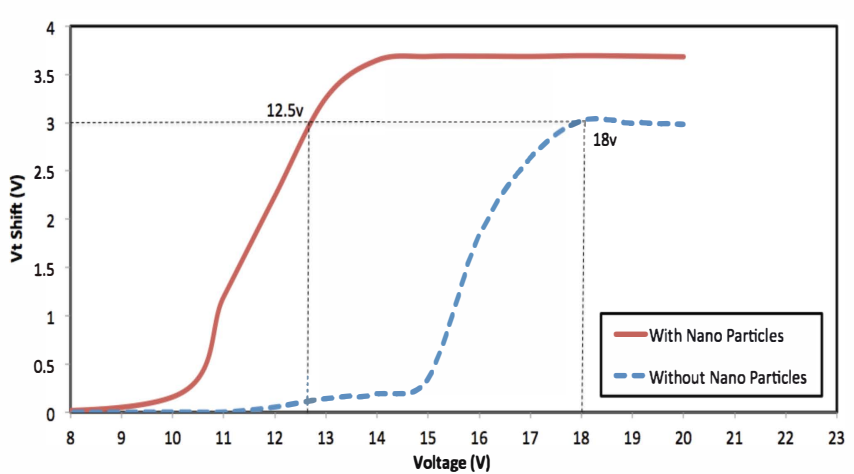

Figure 6: $\mathrm{V}_{\mathrm{t}}$ shift vs. programming voltage (with and without nanoparticles)

\section{EFFECT TUNNEL OXIDE AND TRAPPING LAYER}

\section{a. $V_{t}$ shift}

In charge trapping memory cells, the tunnel oxide and trapping layer thickness are key design pararatmers. The nanoparticles will allow for furthur scaling of the tunnel oxide do to the programming voltage reduction. Figure 7 plots the effect of tunnel oxide thickness on the threshold voltage shift for different program/erase voltages with trapping layer thickness of $2 \mathrm{~nm}$. The plot shows for tunnel oxide thickness larger than $15 \mathrm{~nm}$ no memory effect is observed since no tunneling occurs. As the tunnel oxide is scalled below $15 \mathrm{~nm}$ the $\mathrm{V}_{\mathrm{t}}$ shift increases due to the onset of tunneling. The larger the progamming voltage the larger the electric field and the more tunneling occurs from the $\mathrm{ZnO}$ channel to the trapping layer. Figure 8 plots the effect of trapping layer thickness on the threshold voltage shift. The thicker the trapping layer, the more charge can be trapped and the threshold voltage shift is larger.

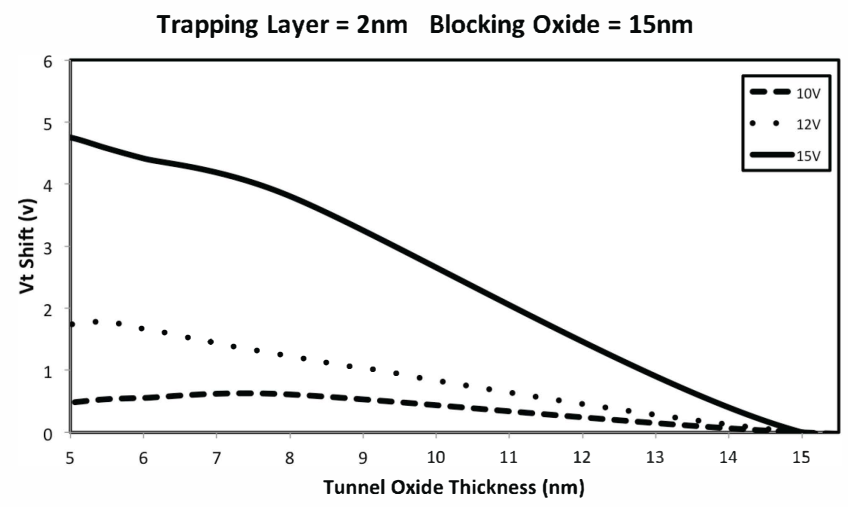

Figure 7: $\mathrm{V}_{\mathrm{t}}$ shift vs. tunnel oxide thickness for different programming voltages

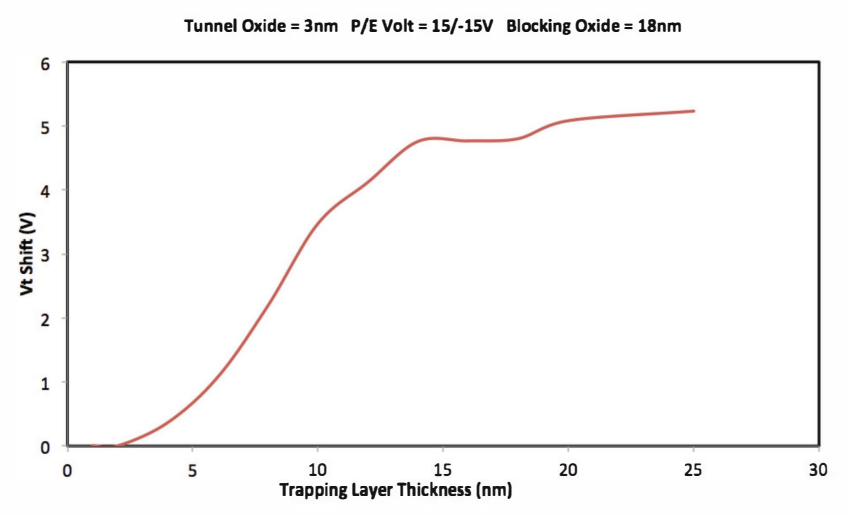

Figure 8: $\mathrm{V}_{\mathrm{t}}$ shift vs. trapping layer thickness

\section{b. Retention}

A 10-year transient simulation is used to model the retention of $\mathrm{ZnO}$ nanoparticle memory. The electron density stored in the traping layer as a function of time, is shown in figure 9 for different tunnel oxide thickness with programming and erasing voltage $=15 /-15 \mathrm{~V}$ and trapping layer thickness of $2 \mathrm{~nm}$. The thicker the tunnel oxide the better retention since less electrons can tunnel out and less holes can tunnel into the trapping layer. The nanoparticles allow for furthur scaling of the tunnel oxide without sacraficing on retention

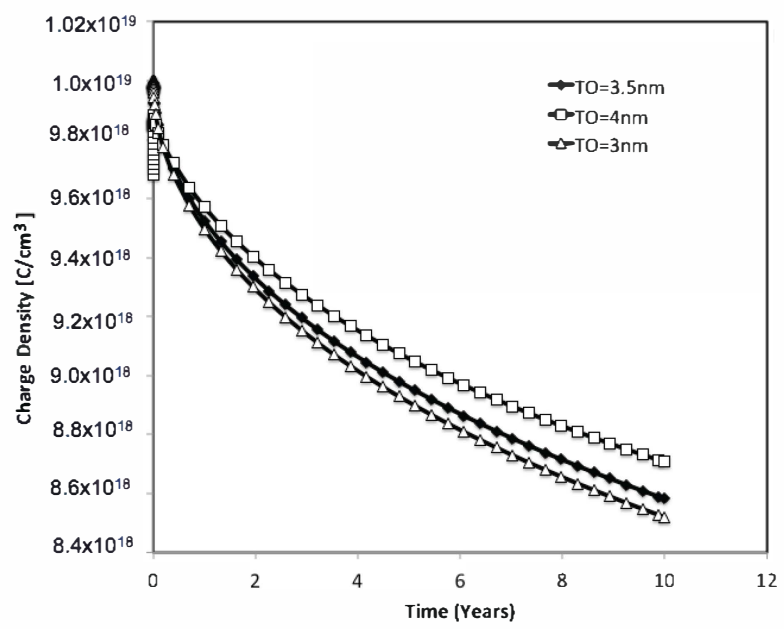

Figure 9: Electron and hole charge densities as a function of time for different tunnel oxide thickness

\section{SUMMARY}

A nanoparticle enhanced thin film $\mathrm{ZnO}$ charge trapping memory cell is demonstrated. The nanoparticles increase the $\mathrm{V}_{\mathrm{t}}$ shift for the same programming voltage. In addition, the nanoparticles reduce the programming voltage required. Finally these results show that nanoparticles can be used to improve current memory technologies and offer an exciting path for future nano-memories 


\section{ACKNOWLEDGMENT}

We gratefully acknowledge financial support for this work provided by the Masdar Institute of Science and Technology.

\section{REFERENCES}

[1] R. Ohba, N. Sugiyama, K. Uchida, J. Koga, and A. Toriumi, "Non- volatile Si quantum memory with self-aligned doublystacked dots," IEEE Trans. Electron Devices, vol. 49, no. 8, pp. 1392-1398, Aug. 2002.

[2] M. Takata, S. Kondoh, T. Sakaguchi, H. Choi, J.-C. Shim, H. Kurino, and M. Koyanagi, "New nonvolatile memory with extremely high density metal nano-dots," in IEDM Tech. Dig., 2003, pp. 553-557.

[3] J. De Blauwe, "Nanocrystal Nonvolatile Memory Devices," IEEE Transactions On Nanotechnology, vol. 1, no. 1, March 2002

[4] C. gyu Hwang 2 "Nanotechnology enables a new memory growth model," Proceedings Of The IEEE, vol. 91, no. 11, November 2003

[5] D. Gupta, M. Anand, S.W. Ryu, Y.K. Choi, and S. Yoo, "Nonvolatile memory based on sol-gel $\mathrm{ZnO}$ thin-film transistors with $\mathrm{Ag}$ nanoparticles embedded in the $\mathrm{ZnO} /$ gate insulator interface", Appl. Phys. Lett., vol. 93, no. 22, 2008.

[6] H. Yin, S. Kim, C. J. Kim, I. Song, J. Park, S. Kim, and Y. Park, "Fully transparent nonvolatile memory employing amorphous oxides as charge trap and transistor's channel layer,"Appl. Phys. Lett., vol. 93, no. 17, October 2008.

[7] S. Kang, Y. Kim, H. S. Seo,S. W. Son, E. A. Yoon, S. Joo and C. W. Ahn, "High-performance and room-temperature-processed nanofloating gate memory devices based on top-gate transparent thin-film transistors," Appl. Phys. Lett., vol. 98, no. 21, May 2011.

[8] E. Kim, Y. Kim, D. H. Kim, K. Lee, G.N. Parsons, and K. Park, "SiNx charge-trap nonvolatile memory based on $\mathrm{ZnO}$ thin-film transistors," Appl. Phys. Lett., vol. 99, 2011.

[9] Y. T. Shih, M. K. Wu, M. J. Chen, Y. C. Cheng, J. R. Yang, And M. Shiojiri, "ZnO-based heterojunction light-emitting diodes on $\mathrm{p}-\mathrm{SiC}(4 \mathrm{H})$ grown by atomic layer deposition," Applied Physics B - Lasers and Optics, vol. 98, 2010

[10] M. L. Huang, Y. C. Chang, C. H. Chang, T. D. Lin, J. Kwo, T. $\mathrm{B}$. $\mathrm{Wu}$, And $\mathrm{M}$. Hong, "Energy-band parameters of atomiclayer-deposition $\mathrm{Al}_{2} \mathrm{O}_{3} / \mathrm{InGaAs}$ heterostructure," Appl. Phys. Letter, vol. 89, 2006.

[11] S. J. Pearton, D. P. Norton, K. Ip,Y.W. Heo, T. Steiner, "Recent progress in processing and properties of $\mathrm{ZnO}$," Prog. Mater. Sci., vol. 50, no. 3, pp. 293-340, 2005.

[12] M. Specht, M. Städele, S. Jakschik, and U. Schröder, "Transport mechanisms in atomic-layer-deposited $\mathrm{Al}_{2} \mathrm{O}_{3}$ dielectrics," Appl. Phys. Lett., vol. 84, no. 16, 2001.

[13] P. Vitanov, A. Harizanova, T. Ivanova, T. Dimitrova, "Study of $\mathrm{ZrO}_{2} / \mathrm{Al}_{2} \mathrm{O}_{3} / \mathrm{ZrO}_{2}$ and $\mathrm{Al}_{2} \mathrm{O}_{3} / \mathrm{ZrO}_{2} / \mathrm{Al}_{2} \mathrm{O}_{3}$ Stack Structures Deposited by Sol-Gel Method on Si," IOP Conf. Ser.: Mater. Sci. Eng. 8, 2010.

[14] Yee-Chia Yeo,Tsu-Jae King and Chenming Hu, "Metaldielectric band alignment and its implications for metal gate complementary metal-oxide-semiconductor technology," Journal of Applied Physics, vol. 92, no. 12, 2002.

[15] V. Rose and R. Franchy, "The band gap of ultrathin amorphous and well-ordered $\mathrm{Al}_{2} \mathrm{O}_{3}$ films on $\mathrm{CoAl}(100)$ measured by scanning tunneling spectroscopy," Journal of Applied Physics, vol. 105 , no. 72009.

[16] J. Bu and M.H. White, "Design considerations in scaled SONOS nonvolatile memory devices," Solid-State Electronics, vol. 45, no. 1, pp. 113-120, 2001.

[17] P. Chakraborty, S. S. Mahato, T. K. Maiti, S. Saha and C K Maiti, "Nanocrystal Non-Volatile Flash Memory Devices: A Simulation Study," NateHCA, IETE Mumbai Centre, 2007. 